\title{
Pre-Messinian (Sub-Salt) Source-Rock Potential on Back-Stop Basins of the Hellenic Trench System (Messara Basin, Central Crete, Greece)
}

\author{
A. Maravelis ${ }^{1 *}$, G. Panagopoulos ${ }^{2}$, I. Piliotis ${ }^{3}$, N. Pasadakis ${ }^{4}$, E. Manoutsoglou ${ }^{2}$ \\ and A. Zelilidis ${ }^{3}$ \\ 1 School of Environmental and Life Sciences, University of Newcastle, Callaghan 2308 NSW - Australia \\ ${ }^{2}$ Laboratory of Geology, Department of Mineral Resources Engineering, Technical University of Crete, Chania - Greece \\ ${ }^{3}$ Laboratory of Sedimentology, Department of Geology, University of Patras - Greece \\ ${ }^{4}$ Hydrocarbon Chemistry and Technology Research Unit, Department of Mineral Resources Engineering, Technical University of Crete, \\ Chania - Greece \\ e-mail: angelos.maravelis@newcastle.edu.au \\ * Corresponding author
}

\begin{abstract}
Résumé - Roche-mère potentielle du Messinien pré-évaporitique d'un basin adossé à l'ensemble de fosses hellèniques (Messara, Crète central, Grèce) - Le secteur grec de la Ride Méditerranéenne, $\mathrm{vu}$ son potentiel en hydrocarbures, demande une exploration frontalière prononcée. Les similitudes géologiques entre ses parties prolifiques, incluses dans les zones économiques exclusives chypriote et égyptienne, indiquent des réserves possibles et récupérables de gaz naturel dans la partie de la zone économique exclusive grecque. Néanmoins, et jusqu'à présent, il manque une étude frontalière systématique, bien que des indices directs pétroliers existent. Plusieurs volcans de boues actives sur la Ride Méditerranéenne, émettant constamment du gaz et des hydrates de gaz, n'ont pas encore été étudiés, quoiqu'en général, dans le monde entier, ils sont fortement liés à la présence d'hydrocarbures (par exemple dans la Mer Caspienne, le Golfe du Mexique, le Bassin Africain Occidental, à Trinité-et-Tobago et dans le cône du Nil). Pour cette raison, la roche mère potentielle dans les successions sédimentaires du Miocène Supérieur d'un bassin 'back-stop' du système du Sillon Hellénique (bassin de Messara, Crète centrale, Grèce), a été étudiée. Les données de la pyrolyse indiquent que la teneur en matière organique est suffisante et avec une qualité assez bonne pour la considérer comme une roche mère potentielle. En outre, le type du kérogène III, suggère un potentiel de genèse de gaz.

Même si des indications d'une évolution thermique plus élevée existent, les roches étudiées témoignent d'un faible niveau de maturation. Des suintements de gaz biogénique dans la région étudiée démontrent le potentiel régional de génération de gaz.
\end{abstract}

\begin{abstract}
Pre-Messinian (Sub-Salt) Source-Rock Potential on Back-Stop Basins of the Hellenic Trench System (Messara Basin, Central Crete, Greece) - The Greek part of the Mediterranean Ridge suggests, in terms of its hydrocarbon potential, further frontier exploration. The geological similarities between its prolific portions, within the Cyprus and Egyptian Exclusive Economic Zones, indicate possible recoverable natural gas reserves in its Greek portion. Nevertheless it lacks of
\end{abstract}


systematic frontier exploration although direct petroleum indicators occur. Active mud volcanoes on the Mediterranean Ridge, still emitting concurrently gas and gas hydrates, have not been yet assessed even though are strongly related to hydrocarbon occurrence worldwide (Caspian Sea, Gulf of Mexico, Western African Basin, Trinidad-Tobago, the Nile Cone). For this reason, the source rock potential of the Late Miocene lacustrine deposits on a backstop basin of the Hellenic Trench System (Messara Basin, Crete, Greece), was studied. The obtained pyrolysis data indicate that the containing organic matter is present in sufficient abundance and with good enough quality to be regarded as potential source rocks. The observed type III kerogen suggests gas generation potential. Although indications of higher thermal evolution occur the studied rocks suggest low maturation levels. The biogenic gas seeps in the studied research well further demonstrate the regional gas generation potential.

\section{INTRODUCTION}

Even though the demand in oil and gas is growing worldwide, Greece's potential in this sector still remains poorly explored. The country recently, opened up a little to the foreign investments and such willingness may entice many international oil and gas exploration companies to compete for acreage. The Hellenic Republic, through its Competent Authority, the Ministry of Environment, Energy and Climate Change, invites (open door) qualified natural or legal persons or any group of such persons to apply for granting and using authorizations for exploration and exploitation of hydrocarbon in respect of certain acreage in Western Greece. The Ministry of Environment, Energy and Climate Change additionally invited companies to submit applications for participation in a non-exclusive seismic survey offshore Western and Southern Greece.

From southwest Peloponnesus to south of Crete and Rhodes, the eastern Mediterranean seafloor is characterized by a large, arc-shaped, sedimentary wedge, known as the Mediterranean Ridge (MR). This ridge is more than $1500 \mathrm{~km}$ long and 200-250 km wide (Heezen and Ewing, 1963; Emery et al., 1966). Its formation results from the subduction of the African plate beneath Eastern Europe (Olivet et al., 1982; Reillinger et al., 1997; Kreemer and Chamot-Rooke, 2004) (Fig. 1). To the north, the MR is bounded by the Hellenic Trench System (HTS) that surrounds the continental slopes of the foot of Peloponnesus, Crete and Rhodes (Huchon et al., 1982). To the south, faces discontinuous segments of abyssal plains whose water depths range between $4000 \mathrm{~m}$ (Ionian Abyssal Plain) to $3100 \mathrm{~m}$ (Herodotus Abyssal Plain) with only a very narrow, flat-bottomed furrow (water depth averaging $2800 \mathrm{~m}$ ) north of the steep Libyan continental slope (Huguen and Mascle, 2001).

The use of new geophysical exploration techniques applied in deep offshore (drilling beyond 3000 meters of water and 3D seismic and reservoir characterization), paved the way in developing and producing hydrocarbons in new, promising areas of the Eastern Mediterranean (Bruneton et al., 2011). Several discoveries have been reported within the Levantine Basin while another $1.3 \mathrm{tcm}$ offshore Cyprus may exists (Gardosh et al., 2009; Semb, 2009). Herodotus Basin partly lies in the Greek Exclusive Economic Zone (EEZ) and appears to have significant oil/gas potential including probable large stratigraphic traps and working petroleum systems. Direct hydrocarbon indications such as active mud volcanoes have been observed on seismic data (Montadert and Nikolaides, 2007; Krois et al., 2010). Recently, substantial oil and gas field discoveries offshore Israel were yielded in a similar to Cyprus offshore geological environment. Similar discoveries offshore Egypt (Nile Cone), adjoining the Greek EEZ, can further strengthen the probability of discovering similar reserves into the Greek offshore. Many of the depressions in the uppermost sector of the MR are considered to have formed by dissolution of the Miocene Messinian evaporite layers and they have become anoxic basins (Camerlenghi and McCoy, 1990; Cita, 1991). These depressions are located on the inner tectonic unit that forms the 'backstop' of the Ridge (Winckler et al., 1997). Seven potential hydrocarbon plays have been proposed in the Greek part of the MR (Maravelis et al., 2012). The most promising areas are seen offshore south, southeast and southwest of Crete, offshore NW Peloponnesus and Herodotus Basin (Maravelis et al., 2012).

A great opportunity to advance our understanding on the hydrocarbon potential onshore Crete and adjoining area is offered by the Late Miocene (Tortonian-Early Messinian) sedimentary rocks on Messara Basin, Central Crete. Establishment of sub-salt sediments (preMessinian) with source rock and/or reservoir potential is crucial since the evaporites may potentially serve as a first class seal immediately below the base of the salt. A vast salt deposit beneath the floor of the Mediterranean has been noticed at the time of the first deep-sea drilling in 1970 (Hersey, 1965; Ryan et al., 1971). The southern dipping of the studied rock indicates that the 


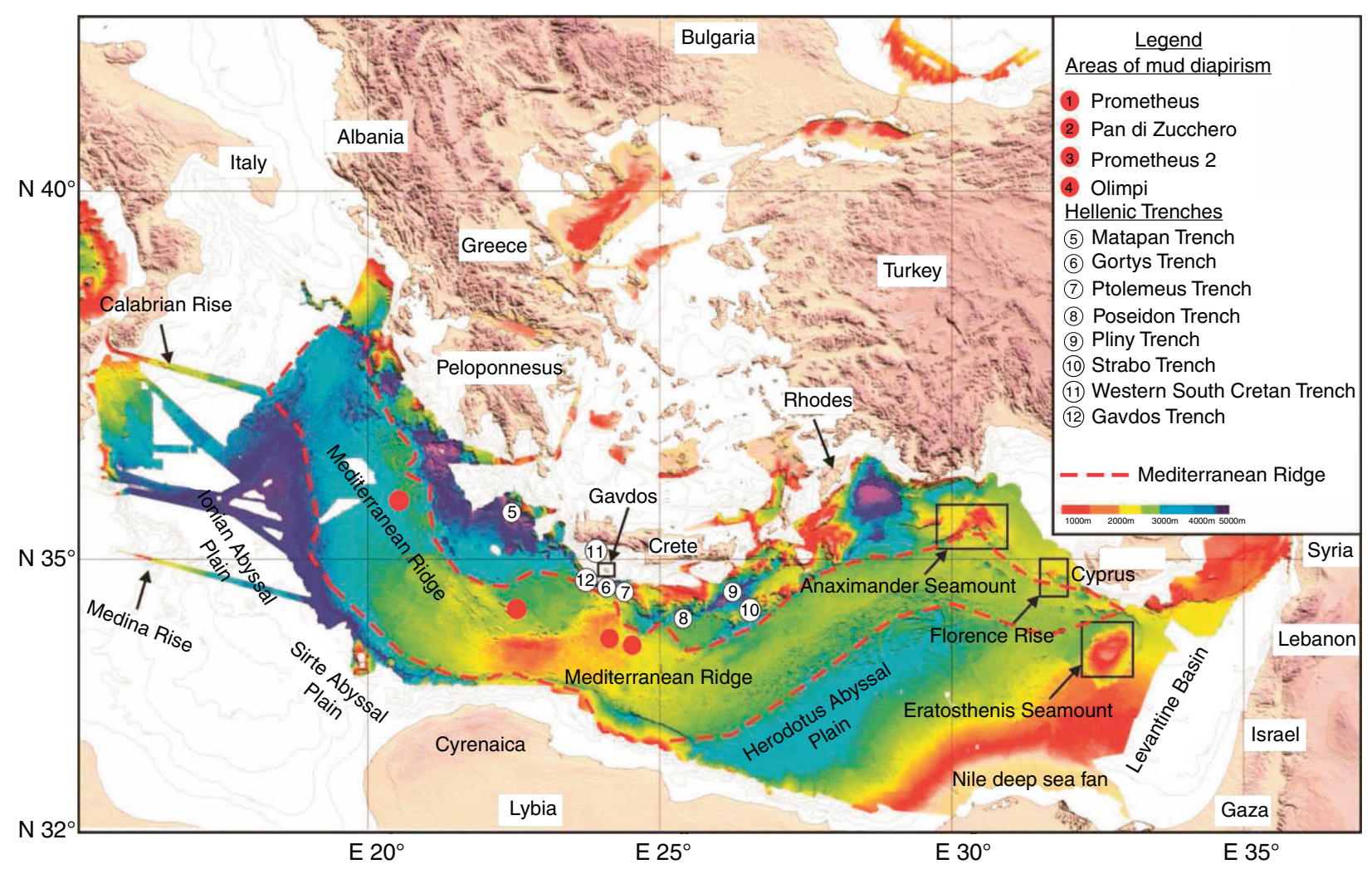

Figure 1

Position of the Mediterranean Ridge in the Eastern Mediterranean Sea and related structural elements (modified from Maravelis et al., 2012).

main boundary fault is located at the southern basin margin (Panagopoulos et al., 2011a). Thus, the thickness of the Neogene deposits increases southward, where the muddy layers of studied sediments may have the maximum extent. Turbidity currents may have contributed significant amount of coarse-grained detritus to the deepest parts forming submarine fans. In that case, thick sand lobes could act as potential $\mathrm{H} / \mathrm{C}$ reservoirs (Panagopoulos et al., 2011a). Furthermore, the occurrence of syn-sedimentary faults favors the lateral restriction of sand deposits and the establishment of vertical connection through faults (Panagopoulos et al., 2011a). The southern dipping of bedding favors northward directed gas migration, in the shallower sand deposits (Panagopoulos et al., 2011a).

This research is the first detailed organic geochemical study of these rocks and attempts to provide original pyrolysis data to improve our understanding of the hydrocarbon potential on this high prospectivity area. During this project four exploration wells were drilled and gas seepages were observed. Gas composition, was approximately $90 \%$ methane and $10 \%$ nitrogen, indicating a possible biogenic origin. Well $\mathrm{D}$ exhibits the greater gas seepage and is the focus of the current research.

\section{GEOLOGICAL SETTING}

The Aegean region is one of the best-studied continental extensional provinces in the world. The continental crust of the Aegean consists of a stack of upper crustal nappes (Jacobshagen, 1986; Faccenna et al., 2003). Such nappes accreted during northward dipping, long-lived and stillactive subduction that accommodated Africa-Europe convergence since the Cretaceous (Jacobshagen, 1986; Faccenna et al., 2003; Van Hinsbergen et al., 2005). As consequences of plate movements, the Alpidic mountain chains were up-folded, the marine connections to the Indo-Pacific and Paratethys were interrupted, and the marine passage to the Atlantic Ocean became continuously restricted (Rögl and Steininger, 1984). 
Hence, during the late Miocene nearly the present-day configuration of the Mediterranean had formed (Rögl and Steininger, 1984). An important driver for the changes in landscapes and environments in the eastern Mediterranean during the Neogene was the rollback of the Hellenic Subduction Zone since the late Oligocene (Ten Veen and Meijer, 1998; Fassoulas, 2001; Van Hinsbergen et al., 2005; Tortorici et al., 2010). Until this time Crete, as part of the Aegean plate, was connected with the European continent (Rögl and Steininger, 1984; Meulenkamp et al., 1994).

The island of Crete represents a prominent horst structure formed in the recent fore-arc of the still ongoing Hellenic subduction. The structure of Crete consists of a pile of nappes that contain rock units from various paleogeographical zones that could be separated coarsely into two categories. The lower Unit of HP-LT metamorphic rocks and the upper Unit of non-metamorphic rocks (Van Hinsbergen and Meulenkamp, 2006; Papanikolaou and Vassilakis, 2010; Zachariasse et al., 2011). The general belief is that the tectonic contact of these two units is an extensional detachment striking E-W, the so-called Cretan Detachment. This zone acted since Middle Miocene (15-17 Ma) as a low angle normal fault and is observed in Crete as well as in Cyclades (Ring et al., 2001; Van Hinsbergen and Meulenkamp, 2006; Tortorici et al., 2010).

The Neogene basins of Crete formed in response to extensional geodynamic processes in the Aegean region during the late Middle Miocene (Fassoulas, 2001; Meulenkamp and Sissingh, 2003). Subsidence occurred in complex half-graben systems in a variety of larger and smaller basins being separated by pronounced uplifts (Ten Veen and Postma, 1999). Although subsequent faulting blurred original facies architectures, present-day distribution of basins and uplifts in central Crete still shows some reflections of Late Miocene palaeogeography (Ten Veen and Postma, 1999; Reuter et al., 2006). Large uplifted blocks composed of pre-Neogene sediments (limestones, sandstones, mudstones), metamorphites (phyllite, quartzite) and ultramafic rocks (ophiolites) separate these basins and form the present-day mountains of Crete (e.g., Ida Mountains, Asteroussia Mountains) (Ten Veen and Postma, 1999). During the Late Miocene these blocks formed islands undergoing erosion while within the basins siliciclastic fluviatile, brackish and marine sediments were accumulated (Reuter et al., 2006; Reuter and Brachert, 2007).

The uplift and exhumation of the basement was accompanied by structural disintegration of the hanging wall, which led to the formation of sedimentary basins on top of the nappe pile in the Lower to Middle Miocene. Messara basin was developed over the hanging wall of Cretan detachment forming a supra-detachment basin with deformed basement and several extensional klippen (Van Hinsbergen and Meulenkamp, 2006). The sedimentary characteristics of Neogene basins reflect the tectonic regime of the Hellenic fore-arc at that time. Neogene deposits in Messara basin have undergone multidirectional extensional tectonic events as well as compressional events (Van Hinsbergen and Meulenkamp, 2006; Chatzaras et al., 2006; Tortorici et al., 2010). The tectonic regime since $11 \mathrm{Ma}$ is explained by an arc-normal pull which caused radial normal faulting (Ten Veen and Meijer, 1998). Later, the arc-normal pull co-existed with transform resistance in the Pliny and Strabo trenches which activated additionally a strike-slip and oblique faulting (Ten Veen and Meijer, 1998).

The Messara Basin is one of the largest Neogene sedimentary basins of Crete. Is bordered by the Ida Mountains to the northwest, the Dicti Mountains to the northeast and the Asteroussia Mountains to the southeast (Fig. 2). A transition in nearshore environments from siliciclastic to carbonate sedimentation in the course of the Tortonian is observed (Angelier et al., 1982; Postma et al., 1993; Ten Veen and Postma, 1999; Reuter et al., 2006). Such transition is accompanied by increasing fragmentation of the basin by normal faulting (Angelier et al., 1982; Ten Veen and Postma, 1999). During the Neogene, conglomerates, sandstones, mudstones, marls, carbonates and evaporates were accumulated in Messara Basin (Zachariasse et al., 2011).

\section{STRATIGRAPHY}

So far, no formal lithostratigraphic subdivision was available for the Miocene deposits of central Crete. Most authors applied the preliminary scheme of Meulenkamp et al. (1979), in terms of four formations, namely Viannos, Skinias, Ambelouzos and Varvara Formations. This subdivision was inadequate to fully portray the geological history of the Messara Basin and thus, Zachariasse et al. (2011) has upgraded and formalized both stratigraphy and geochronology. This new classification scheme subdivides the upper Miocene depositional succession of central Crete in six formations. Such formations, namely Viannos, Skinias, Kasteliana, Moulia, Agios Miron and Chersonisos Formations, are timely constrained in the Tortonian-upper Messinian. In the current research, the already broadly applied nomenclature of Meulenkamp et al. (1979) and the classification scheme of Zachariasse et al. (2011) are adopted to control the different environment of deposition (Fig. 3).

The studied sedimentary rocks comprise alternations of mudstones, sandstones, loose sands and conglomerates. Viannos Fm overlies the pre-Neogene basement 


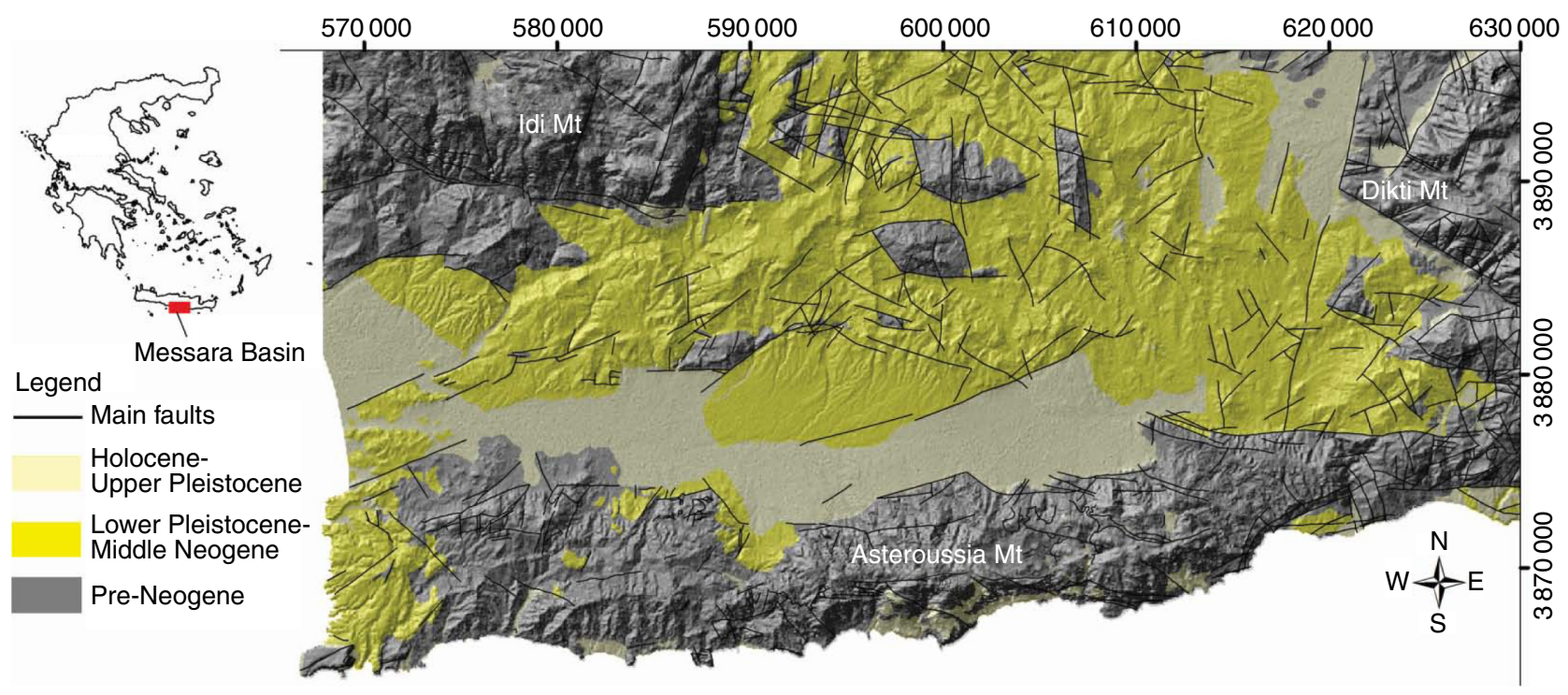

Figure 2

Surface extent of Neogene deposits in the Messara Basin according to geological mapping of Geological Survey of Greece (IGME) in scale of 1:50 000. The faulted boundaries of the study area between Idi Mt. and Asteroussia Mt. directions and the co-existing EW and NE-SW neotectonic structures are clearly portrayed (modified from Panagopoulos et al., 2011b).

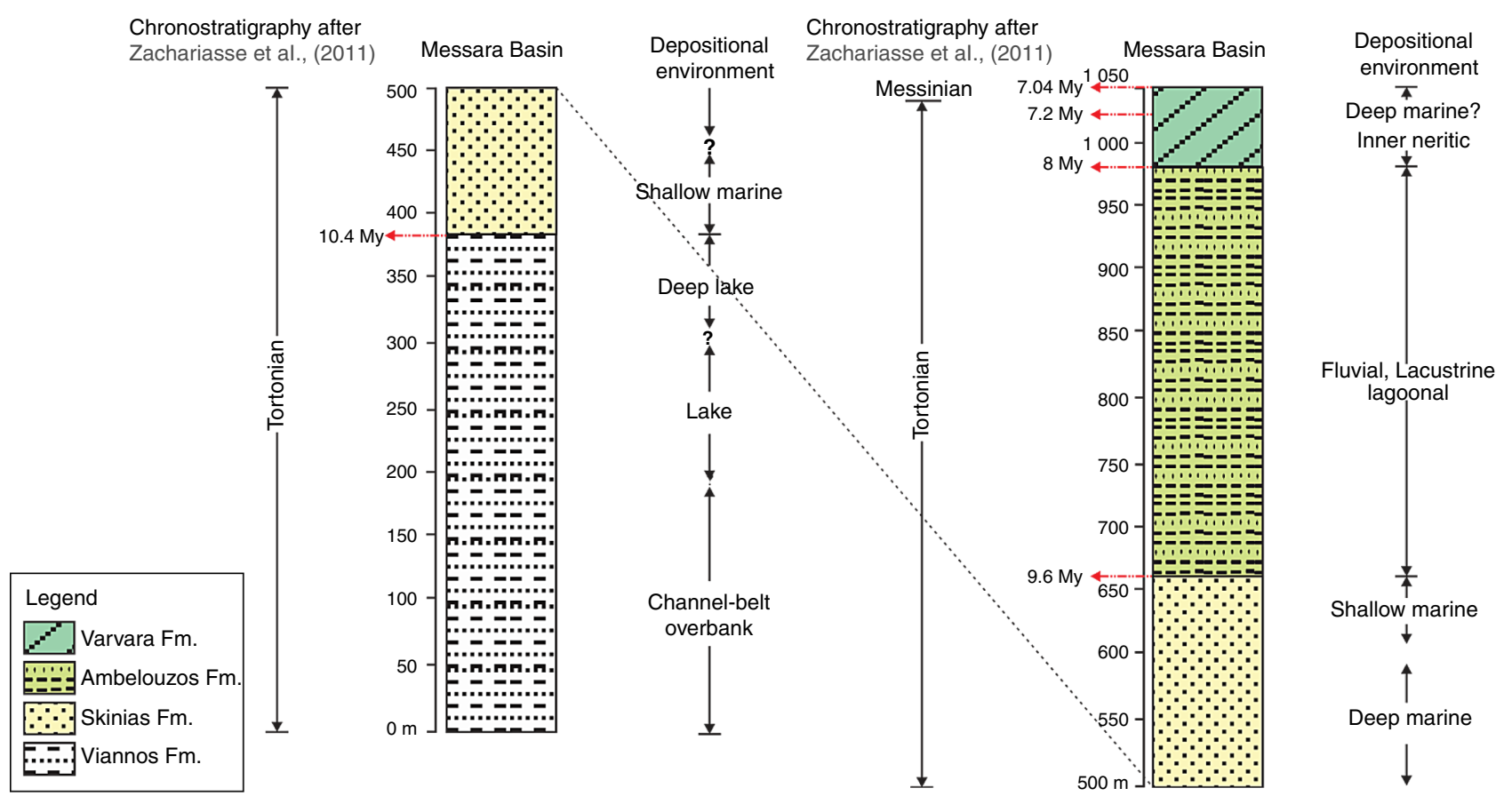

Figure 3

Chronostratigraphy, depositional environments and age model of Tortonian-Early Messinian sedimentary rocks, Messara Basin, Central Crete, with time scale (from Zachariasse et al., 2011). 


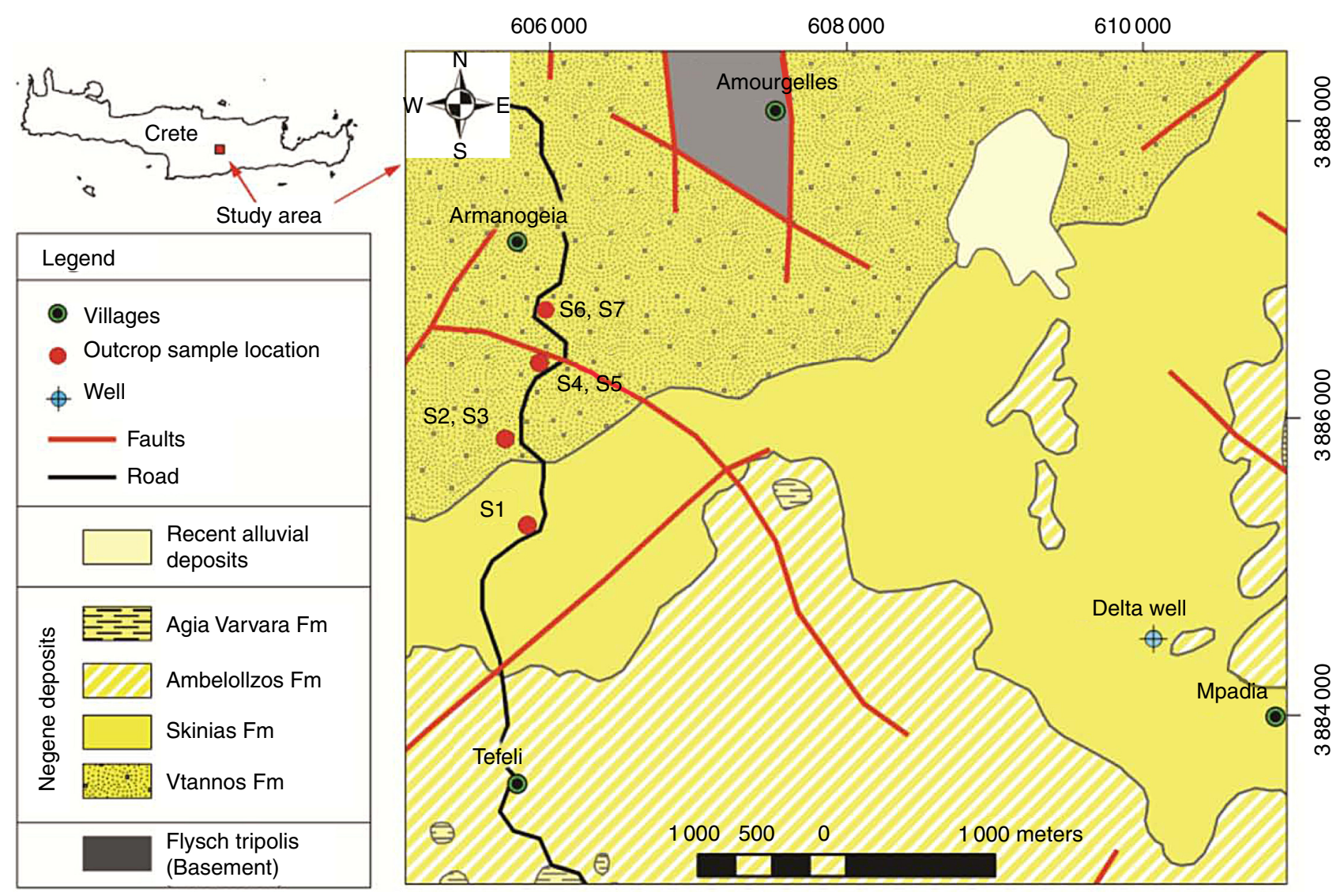

Figure 4

Simplified geological sketch map of study area (modified from Panagopoulos et al., 2011b).

(Fig. 3, 4). Coarse, angular debris occur at the contact surface (Zachariasse et al., 2011). Viannos Fm comprises a wide range of depositional environments such as, channel-belt, overbank and lake deposits. The breccioconglomerates contain sub-angular and unsorted components (Zachariasse et al., 2011). These features suggest that the environment of deposition was an alluvial fan with sheet-flood deposition (Zachariasse et al., 2011). Skinias Fm conformably overlies the Viannos Fm (Fig. 3, 4). Such Formation consists of marine clays with interbedded sandstones and occasional gravels (Zachariasse et al., 2011). Gravity flow deposits such as, slumps and/or graded pebbly mudstones are rare (Zachariasse et al., 2011). Viannos Fm was deposited within a large lake and influenced by ENE-WSW striking faults. The mean grain size decreases southward and suggests a northern main source of sediments (Panagopoulos et al., 2011b). The prevalence of Middle Miocene WNW-ESE striking faults further contributed to the opening of the Viannos Lake and its connection with sea during the deposition of Skinias Fm (Panagopoulos et al., 2011b).

\section{SAMPLES AND METHODS}

A total of 119 samples were selected and the Rock-Eval and TOC data are summarized in Table 1. Organic geochemistry research was performed on samples from both cores (112) and outcrops (7). Core samples were obtained during drilling of the well $\mathrm{D}$ while outcrop samples (S1 to S7) were selected by colour for total organic carbon and Rock-Eval pyrolysis analyses.

Both well and outcrop samples prior to the analysis, were thoroughly washed with distilled water to remove possible contamination from the mud (water-based) used during drilling and dried at $100^{\circ} \mathrm{C}$. All samples were analyzed in a Rock-Eval II (Delsi Inc.) analyzer following the typical experimental procedure of Espitalié et al. (1977). Detailed description of the Rock-Eval analytical 
TABLE 1

Results of Rock-Eval 2 pyrolysis of the selected well (D0-6 to D445-450) and outcrop ( $S 1$ to S7) samples (TOC: Total Organic Carbon, PI: Production Index, HI: Hydrogen Index, OI: Oxygen Index)

\begin{tabular}{|c|c|c|c|c|c|c|c|c|c|c|c|c|c|}
\hline Sample & $\begin{array}{c}\text { Depth } \\
\text { (m) }\end{array}$ & $\begin{array}{c}\text { Mass } \\
(\mathrm{g})\end{array}$ & $\operatorname{Tmax}$ & S1 & $S 2$ & $S 3$ & TOC & $P I$ & $S 1+S 2$ & $S 2 / S 3$ & $H I$ & $O I$ & $S 1 / T O C$ \\
\hline D0-6 & 0.00 & 93.2 & 0 & 0.0 & 0.00 & 0.16 & 0.12 & 0 & 0.00 & 0.00 & 0 & 133 & 0.00 \\
\hline D6-14 & 10.0 & 94.6 & 408 & 0.04 & 0.40 & 0.61 & 0.62 & 0.09 & 0.44 & 0.66 & 65 & 98 & 0.06 \\
\hline D14-17 & 15.5 & 92.6 & 412 & 0.03 & 0.41 & 0.56 & 0.64 & 0.07 & 0.44 & 0.73 & 64 & 88 & 0.05 \\
\hline D17-23 & 20.0 & 91.6 & 411 & 0.03 & 0.38 & 0.63 & 0.62 & 0.07 & 0.41 & 0.60 & 61 & 102 & 0.05 \\
\hline D23-28 & 25.5 & 91.0 & 411 & 0.01 & 0.18 & 0.49 & 0.34 & 0.05 & 0.19 & 0.37 & 53 & 144 & 0.03 \\
\hline D28-31 & 29.5 & 89.7 & 413 & 0.02 & 0.20 & 0.55 & 0.36 & 0.09 & 0.22 & 0.36 & 56 & 153 & 0.06 \\
\hline D31-35 & 33.0 & 92.9 & 413 & 0.02 & 0.16 & 0.44 & 0.35 & 0.11 & 0.18 & 0.36 & 46 & 126 & 0.06 \\
\hline D35-38 & 37.00 & 89.9 & 417 & 0.01 & 0.14 & 0.62 & 0.36 & 0.07 & 0.15 & 0.23 & 39 & 172 & 0.03 \\
\hline D38-45 & 41.5 & 91.8 & 414 & 0.02 & 0.17 & 0.66 & 0.33 & 0.11 & 0.19 & 0.26 & 52 & 200 & 0.06 \\
\hline D45-50 & 47.5 & 90.9 & 414 & 0.03 & 0.19 & 0.70 & 0.35 & 0.14 & 0.22 & 0.27 & 54 & 200 & 0.09 \\
\hline D50-53 & 51.5 & 91.3 & 422 & 0.01 & 0.16 & 0.61 & 0.36 & 0.06 & 0.17 & 0.26 & 44 & 169 & 0.03 \\
\hline D53-57 & 55.0 & 84.7 & 395 & 0.07 & 0.28 & 0.57 & 0.37 & 0.20 & 0.35 & 0.49 & 76 & 154 & 0.19 \\
\hline D57-60 & 58.5 & 92.8 & 414 & 0.02 & 0.24 & 0.60 & 0.39 & 0.08 & 0.26 & 0.40 & 62 & 154 & 0.05 \\
\hline D60-64 & 62.0 & 96.1 & 412 & 0.02 & 0.24 & 0.57 & 0.43 & 0.08 & 0.26 & 0.42 & 56 & 133 & 0.05 \\
\hline D64-70 & 67.0 & 94.7 & 408 & 0.03 & 0.26 & 0.60 & 0.42 & 0.10 & 0.29 & 0.43 & 62 & 143 & 0.07 \\
\hline D70-73 & 71.5 & 94.5 & 410 & 0.02 & 0.21 & 0.66 & 0.39 & 0.09 & 0.23 & 0.32 & 54 & 169 & 0.05 \\
\hline D73-77 & 75.0 & 88.9 & 407 & 0.02 & 0.21 & 0.59 & 0.40 & 0.09 & 0.23 & 0.36 & 53 & 148 & 0.05 \\
\hline D77-80 & 78.5 & 90.3 & 415 & 0.02 & 0.27 & 0.65 & 0.46 & 0.07 & 0.29 & 0.42 & 59 & 141 & 0.04 \\
\hline D80-83 & 81.5 & 93.3 & 416 & 0.02 & 0.30 & 0.50 & 0.51 & 0.06 & 0.32 & 0.60 & 59 & 98 & 0.04 \\
\hline D83-87 & 85.0 & 93.6 & 411 & 0.02 & 0.21 & 0.49 & 0.43 & 0.09 & 0.23 & 0.43 & 49 & 114 & 0.05 \\
\hline D87-91 & 89.0 & 89.8 & 414 & 0.02 & 0.22 & 0.59 & 0.39 & 0.08 & 0.24 & 0.37 & 56 & 151 & 0.05 \\
\hline D91-95 & 93.0 & 94.0 & 415 & 0.02 & 0.23 & 0.62 & 0.41 & 0.08 & 0.25 & 0.37 & 56 & 151 & 0.05 \\
\hline D95-100 & 97.5 & 90.4 & 416 & 0.02 & 0.28 & 0.60 & 0.52 & 0.07 & 0.30 & 0.47 & 54 & 115 & 0.04 \\
\hline D100-104 & 102.0 & 92.3 & 415 & 0.01 & 0.20 & 0.89 & 0.34 & 0.05 & 0.21 & 0.22 & 59 & 262 & 0.03 \\
\hline D104-108 & 106.0 & 92.2 & 416 & 0.02 & 0.21 & 0.86 & 0.34 & 0.09 & 0.23 & 0.24 & 62 & 253 & 0.06 \\
\hline D108-112 & 110.0 & 92.7 & 408 & 0.03 & 0.24 & 0.87 & 0.39 & 0.11 & 0.27 & 0.28 & 62 & 223 & 0.08 \\
\hline D112-116 & 114.0 & 96.5 & 410 & 0.02 & 0.24 & 0.82 & 0.40 & 0.08 & 0.26 & 0.29 & 60 & 205 & 0.05 \\
\hline D116-120 & 118.0 & 88.5 & 412 & 0.02 & 0.21 & 0.72 & 0.39 & 0.09 & 0.23 & 0.29 & 54 & 185 & 0.05 \\
\hline D120-124 & 122.0 & 91.2 & 416 & 0.02 & 0.19 & 0.77 & 0.39 & 0.10 & 0.21 & 0.25 & 49 & 197 & 0.05 \\
\hline D124-128 & 126.0 & 96.2 & 412 & 0.02 & 0.24 & 0.81 & 0.43 & 0.08 & 0.26 & 0.30 & 56 & 188 & 0.05 \\
\hline D128-132 & 130.0 & 92.4 & 412 & 0.02 & 0.22 & 0.81 & 0.42 & 0.08 & 0.24 & 0.27 & 52 & 193 & 0.05 \\
\hline
\end{tabular}


TABLE 1 (continued)

\begin{tabular}{|c|c|c|c|c|c|c|c|c|c|c|c|c|c|}
\hline Sample & $\begin{array}{l}\text { Depth } \\
(\mathrm{m})\end{array}$ & $\begin{array}{c}\text { Mass } \\
(\mathrm{g})\end{array}$ & $\operatorname{Tmax}$ & $S 1$ & $S 2$ & $S 3$ & TOC & $P I$ & $S 1+S 2$ & $S 2 / S 3$ & $H I$ & $O I$ & $S 1 / T O C$ \\
\hline D132-136 & 134.0 & 92.7 & 411 & 0.03 & 0.29 & 0.75 & 0.48 & 0.09 & 0.32 & 0.39 & 60 & 156 & 0.06 \\
\hline D136-140 & 138.0 & 93.5 & 411 & 0.02 & 0.26 & 0.79 & 0.47 & 0.07 & 0.28 & 0.33 & 55 & 168 & 0.04 \\
\hline D140-144 & 142.0 & 84.6 & 412 & 0.02 & 0.24 & 0.76 & 0.40 & 0.08 & 0.26 & 0.32 & 60 & 190 & 0.05 \\
\hline D144-148 & 146.0 & 90.2 & 412 & 0.02 & 0.23 & 0.77 & 0.38 & 0.08 & 0.25 & 0.30 & 61 & 203 & 0.05 \\
\hline D148-154 & 151.0 & 90.5 & 412 & 0.01 & 0.19 & 0.76 & 0.39 & 0.05 & 0.20 & 0.25 & 49 & 195 & 0.03 \\
\hline D154-158 & 156.5 & 95.4 & 411 & 0.03 & 0.30 & 0.84 & 0.47 & 0.09 & 0.33 & 0.36 & 64 & 179 & 0.06 \\
\hline D159-161 & 160.0 & 94.3 & 412 & 0.03 & 0.28 & 0.83 & 0.41 & 0.10 & 0.31 & 0.34 & 68 & 202 & 0.07 \\
\hline D161-165 & 163.0 & 96.2 & 409 & 0.03 & 0.31 & 0.82 & 0.50 & 0.09 & 0.34 & 0.38 & 62 & 164 & 0.06 \\
\hline D165-169 & 167.0 & 94.6 & 415 & 0.02 & 0.25 & 0.70 & 0.43 & 0.07 & 0.27 & 0.36 & 58 & 163 & 0.05 \\
\hline D169-172 & 170.5 & 91.0 & 416 & 0.04 & 0.23 & 0.78 & 0.37 & 0.15 & 0.27 & 0.29 & 62 & 211 & 0.11 \\
\hline D172-176 & 174.0 & 90.1 & 417 & 0.02 & 0.19 & 0.79 & 0.38 & 0.10 & 0.21 & 0.24 & 50 & 208 & 0.05 \\
\hline D176-180 & 178.0 & 92.3 & 416 & 0.02 & 0.22 & 0.73 & 0.40 & 0.08 & 0.24 & 0.30 & 55 & 183 & 0.05 \\
\hline D180-183 & 181.5 & 92.0 & 414 & 0.02 & 0.22 & 0.71 & 0.43 & 0.08 & 0.24 & 0.31 & 51 & 165 & 0.05 \\
\hline D183-187 & 185.0 & 90.4 & 407 & 0.03 & 0.32 & 0.80 & 0.49 & 0.09 & 0.35 & 0.40 & 65 & 163 & 0.06 \\
\hline D187-191 & 189.0 & 93.2 & 412 & 0.03 & 0.26 & 0.79 & 0.45 & 0.10 & 0.29 & 0.33 & 58 & 176 & 0.07 \\
\hline D191-195 & 193.0 & 96.1 & 410 & 0.03 & 0.30 & 0.71 & 0.46 & 0.09 & 0.33 & 0.42 & 65 & 154 & 0.07 \\
\hline D195-199 & 197.0 & 92.4 & 413 & 0.02 & 0.28 & 0.68 & 0.47 & 0.07 & 0.30 & 0.41 & 60 & 145 & 0.04 \\
\hline D199-203 & 201.0 & 94.6 & 417 & 0.02 & 0.24 & 0.80 & 0.43 & 0.08 & 0.26 & 0.30 & 56 & 186 & 0.05 \\
\hline D203-207 & 205.0 & 90.2 & 415 & 0.01 & 0.23 & 0.64 & 0.42 & 0.04 & 0.24 & 0.36 & 55 & 152 & 0.02 \\
\hline D207-210 & 208.5 & 92.5 & 416 & 0.02 & 0.21 & 0.78 & 0.40 & 0.09 & 0.23 & 0.27 & 53 & 195 & 0.05 \\
\hline D210-214 & 212.0 & 88.9 & 415 & 0.01 & 0.25 & 0.71 & 0.44 & 0.04 & 0.26 & 0.35 & 57 & 161 & 0.02 \\
\hline D214-218 & 216.0 & 93.2 & 416 & 0.02 & 0.25 & 0.72 & 0.45 & 0.07 & 0.27 & 0.35 & 56 & 160 & 0.04 \\
\hline D218-222 & 220.0 & 91.6 & 413 & 0.03 & 0.31 & 0.80 & 0.47 & 0.09 & 0.34 & 0.39 & 66 & 170 & 0.06 \\
\hline D222-226 & 224.0 & 91.9 & 412 & 0.03 & 0.30 & 0.72 & 0.48 & 0.09 & 0.33 & 0.42 & 63 & 150 & 0.06 \\
\hline D226-230 & 228.0 & 92.9 & 411 & 0.02 & 0.29 & 0.72 & 0.45 & 0.06 & 0.31 & 0.40 & 64 & 160 & 0.04 \\
\hline D230-234 & 232.0 & 93.5 & 413 & 0.04 & 0.33 & 0.82 & 0.47 & 0.11 & 0.37 & 0.40 & 70 & 174 & 0.09 \\
\hline D234-238 & 236.0 & 89.7 & 412 & 0.02 & 0.24 & 0.71 & 0.43 & 0.08 & 0.26 & 0.34 & 56 & 165 & 0.05 \\
\hline D238-242 & 240.0 & 96.0 & 410 & 0.02 & 0.28 & 0.72 & 0.43 & 0.07 & 0.30 & 0.39 & 65 & 167 & 0.05 \\
\hline D242-246 & 244.0 & 94.9 & 413 & 0.02 & 0.25 & 0.66 & 0.41 & 0.07 & 0.27 & 0.38 & 61 & 161 & 0.05 \\
\hline D246-250 & 248.0 & 91.3 & 414 & 0.03 & 0.30 & 0.71 & 0.46 & 0.09 & 0.33 & 0.42 & 65 & 154 & 0.07 \\
\hline D250-254 & 252.0 & 92.0 & 408 & 0.02 & 0.30 & 0.67 & 0.48 & 0.06 & 0.32 & 0.45 & 63 & 140 & 0.04 \\
\hline D254-260 & 257.0 & 92.1 & 411 & 0.02 & 0.24 & 0.76 & 0.43 & 0.08 & 0.26 & 0.32 & 56 & 177 & 0.05 \\
\hline
\end{tabular}


TABLE 1 (continued)

\begin{tabular}{|c|c|c|c|c|c|c|c|c|c|c|c|c|c|}
\hline Sample & $\begin{array}{l}\text { Depth } \\
\text { (m) }\end{array}$ & $\begin{array}{c}\text { Mass } \\
(\mathrm{g})\end{array}$ & $\operatorname{Tmax}$ & S1 & $S 2$ & $S 3$ & TOC & $P I$ & $S 1+S 2$ & $S 2 / S 3$ & $H I$ & $O I$ & $S 1 / T O C$ \\
\hline D260-264 & 262.0 & 96.2 & 414 & 0.02 & 0.25 & 0.72 & 0.43 & 0.07 & 0.27 & 0.35 & 58 & 167 & 0.05 \\
\hline D264-268 & 266.0 & 93.2 & 412 & 0.02 & 0.30 & 0.81 & 0.50 & 0.06 & 0.32 & 0.37 & 60 & 162 & 0.04 \\
\hline D268-272 & 270.0 & 91.9 & 416 & 0.02 & 0.28 & 0.76 & 0.46 & 0.07 & 0.30 & 0.37 & 61 & 165 & 0.04 \\
\hline D272-276 & 274.0 & 93.2 & 412 & 0.02 & 0.27 & 0.71 & 0.46 & 0.07 & 0.29 & 0.38 & 59 & 154 & 0.04 \\
\hline D276-280 & 278.0 & 92.0 & 409 & 0.03 & 0.35 & 0.82 & 0.55 & 0.08 & 0.38 & 0.43 & 64 & 149 & 0.05 \\
\hline D280-284 & 282.0 & 89.9 & 411 & 0.02 & 0.31 & 0.83 & 0.51 & 0.06 & 0.33 & 0.37 & 61 & 163 & 0.04 \\
\hline D284-288 & 286.0 & 90.1 & 409 & 0.03 & 0.34 & 0.74 & 0.52 & 0.08 & 0.37 & 0.46 & 65 & 142 & 0.06 \\
\hline D288-292 & 290.0 & 89.9 & 410 & 0.02 & 0.23 & 0.75 & 0.47 & 0.08 & 0.25 & 0.31 & 49 & 160 & 0.04 \\
\hline D292-296 & 294.0 & 95.5 & 409 & 0.03 & 0.31 & 0.77 & 0.49 & 0.09 & 0.34 & 0.40 & 63 & 157 & 0.06 \\
\hline D296-300 & 298.0 & 96.1 & 421 & 0.03 & 0.33 & 0.69 & 0.40 & 0.08 & 0.36 & 0.48 & 83 & 173 & 0.08 \\
\hline D300-304 & 302.0 & 91.8 & 422 & 0.03 & 0.30 & 0.84 & 0.36 & 0.09 & 0.33 & 0.36 & 83 & 233 & 0.08 \\
\hline D304-307 & 305.5 & 93.2 & 420 & 0.03 & 0.31 & 0.69 & 0.40 & 0.09 & 0.34 & 0.45 & 78 & 173 & 0.08 \\
\hline D307-311 & 309.0 & 87.0 & 421 & 0.02 & 0.31 & 0.70 & 0.43 & 0.06 & 0.33 & 0.44 & 72 & 163 & 0.05 \\
\hline D311-314 & 312.5 & 93.9 & 421 & 0.03 & 0.34 & 0.71 & 0.47 & 0.08 & 0.37 & 0.48 & 72 & 151 & 0.06 \\
\hline D314-318 & 316.0 & 93.2 & 416 & 0.02 & 0.30 & 0.64 & 0.24 & 0.06 & 0.32 & 0.47 & 125 & 267 & 0.08 \\
\hline D318-322 & 320.0 & 92.9 & 423 & 0.04 & 0.30 & 0.73 & 0.44 & 0.12 & 0.34 & 0.41 & 68 & 166 & 0.09 \\
\hline D322-327 & 324.5 & 91.9 & 417 & 0.03 & 0.31 & 0.75 & 0.29 & 0.09 & 0.34 & 0.41 & 107 & 259 & 0.10 \\
\hline D327-330 & 328.5 & 92.5 & 412 & 0.03 & 0.30 & 0.58 & 0.33 & 0.09 & 0.33 & 0.52 & 91 & 176 & 0.09 \\
\hline D330-334 & 332.0 & 91.7 & 417 & 0.02 & 0.28 & 0.68 & 0.32 & 0.07 & 0.30 & 0.41 & 88 & 213 & 0.06 \\
\hline D334-338 & 336.0 & 92.3 & 415 & 0.03 & 0.28 & 0.73 & 0.61 & 0.10 & 0.31 & 0.38 & 46 & 120 & 0.05 \\
\hline D338-342 & 340.0 & 94.0 & 414 & 0.03 & 0.26 & 0.71 & 0.41 & 0.10 & 0.29 & 0.37 & 63 & 173 & 0.07 \\
\hline D342-346 & 344.0 & 93.9 & 415 & 0.03 & 0.33 & 0.47 & 0.43 & 0.08 & 0.36 & 0.70 & 77 & 109 & 0.07 \\
\hline D346-350 & 348.0 & 92.7 & 414 & 0.02 & 0.36 & 0.57 & 0.46 & 0.05 & 0.38 & 0.63 & 78 & 124 & 0.04 \\
\hline D350-358 & 354.0 & 92.6 & 416 & 0.03 & 0.44 & 0.71 & 0.55 & 0.06 & 0.47 & 0.62 & 80 & 129 & 0.05 \\
\hline D358-361 & 359.5 & 91.0 & 409 & 0.04 & 0.48 & 0 & 0.55 & 0.08 & 0.52 & 0 & 87 & 0 & 0.07 \\
\hline D360-364 & 362.0 & 93.2 & 421 & 0.03 & 0.36 & 0.65 & 0.43 & 0.08 & 0.39 & 0.55 & 84 & 151 & 0.07 \\
\hline D361-365 & 363.0 & 95.2 & 417 & 0.02 & 0.37 & 0.69 & 0.41 & 0.05 & 0.39 & 0.54 & 90 & 168 & 0.05 \\
\hline D365-369 & 367.0 & 93.8 & 415 & 0.04 & 0.40 & 0.72 & 0.47 & 0.09 & 0.44 & 0.56 & 85 & 153 & 0.09 \\
\hline D369-373 & 371.0 & 91.4 & 413 & 0.03 & 0.37 & 0.68 & 0.43 & 0.08 & 0.40 & 0.54 & 86 & 158 & 0.07 \\
\hline D373-377 & 375.0 & 92.4 & 419 & 0.02 & 0.34 & 0.70 & 0.43 & 0.06 & 0.36 & 0.49 & 79 & 163 & 0.05 \\
\hline D377-380 & 378.5 & 93.0 & 417 & 0.02 & 0.33 & 0.67 & 0.39 & 0.06 & 0.35 & 0.49 & 85 & 172 & 0.05 \\
\hline D380-384 & 382.0 & 95.1 & 413 & 0.03 & 0.34 & 0.70 & 0.45 & 0.08 & 0.37 & 0.49 & 76 & 156 & 0.07 \\
\hline
\end{tabular}


TABLE 1 (continued)

\begin{tabular}{|c|c|c|c|c|c|c|c|c|c|c|c|c|c|}
\hline Sample & $\begin{array}{l}\text { Depth } \\
(\mathrm{m})\end{array}$ & $\begin{array}{c}\text { Mass } \\
(\mathrm{g})\end{array}$ & $\operatorname{Tmax}$ & S1 & $S 2$ & $S 3$ & TOC & $P I$ & $S 1+S 2$ & $S 2 / S 3$ & $H I$ & $O I$ & $S 1 / T O C$ \\
\hline D384-387 & 385.5 & 89.4 & 419 & 0.03 & 0.35 & 0.54 & 0.39 & 0.08 & 0.38 & 0.65 & 90 & 138 & 0.08 \\
\hline D387-391 & 389.0 & 90.2 & 422 & 0.04 & 0.29 & 0.72 & 0.44 & 0.12 & 0.33 & 0.40 & 66 & 164 & 0.09 \\
\hline D391-395 & 393.0 & 65.4 & 420 & 0.01 & 0.36 & 0.67 & 0.47 & 0.03 & 0.37 & 0.54 & 77 & 143 & 0.02 \\
\hline D395-398 & 396.5 & 70.4 & 411 & 0.02 & 0.42 & 0.75 & 0.54 & 0.05 & 0.44 & 0.56 & 78 & 139 & 0.04 \\
\hline D398-402 & 400.0 & 89.3 & 423 & 0.03 & 0.30 & 0.71 & 0.43 & 0.09 & 0.33 & 0.42 & 70 & 165 & 0.07 \\
\hline D402-406 & 404.0 & 80.9 & 423 & 0.01 & 0.29 & 0.56 & 0.46 & 0.03 & 0.30 & 0.52 & 63 & 122 & 0.02 \\
\hline D406-409 & 407.5 & 80.0 & 425 & 0.01 & 0.26 & 0.55 & 0.42 & 0.04 & 0.27 & 0.47 & 62 & 131 & 0.02 \\
\hline D409-413 & 411.0 & 67.5 & 428 & 0.02 & 0.35 & 0.71 & 0.45 & 0.05 & 0.37 & 0.49 & 78 & 158 & 0.04 \\
\hline D413-417 & 415.0 & 77.4 & 422 & 0.03 & 0.40 & 0.67 & 0.50 & 0.07 & 0.43 & 0.60 & 80 & 134 & 0.06 \\
\hline D415-420 & 417.5 & 53.8 & 410 & 0.05 & 0.57 & 0.68 & 0.63 & 0.08 & 0.62 & 0.84 & 90 & 108 & 0.08 \\
\hline D420-424 & 422.0 & 48.4 & 433 & 0.02 & 0.64 & 0.74 & 0.50 & 0.03 & 0.66 & 0.86 & 128 & 148 & 0.04 \\
\hline D424-428 & 426.0 & 58.5 & 483 & 0.03 & 0.41 & 0.68 & 0.42 & 0.07 & 0.44 & 0.60 & 98 & 162 & 0.07 \\
\hline D428-431 & 429.5 & 45.1 & 429 & 0.04 & 0.59 & 0.62 & 0.52 & 0.06 & 0.63 & 0.95 & 113 & 119 & 0.08 \\
\hline D431-435 & 433.0 & 94.0 & 420 & 0.04 & 0.31 & 0.76 & 0.50 & 0.11 & 0.35 & 0.41 & 62 & 152 & 0.08 \\
\hline D435-442 & 438.5 & 44.5 & 490 & 0.02 & 0.58 & 0.58 & 0.48 & 0.03 & 0.60 & 1.00 & 121 & 121 & 0.04 \\
\hline D442-445 & 443.5 & 45.7 & 474 & 0.04 & 0.52 & 0.56 & 0.50 & 0.07 & 0.56 & 0.93 & 104 & 112 & 0.08 \\
\hline D445-450 & 447.5 & 49.7 & 503 & 0.02 & 0.54 & 0.74 & 0.40 & 0.04 & 0.56 & 0.73 & 135 & 185 & 0.05 \\
\hline S1 & & 62.0 & 407 & 0.00 & 0.04 & 0.45 & 0.34 & 0.00 & 0.04 & 0.09 & 12 & 132 & 0.00 \\
\hline$S 2$ & & 65.4 & 450 & 0.00 & 0.04 & 0.19 & 0.25 & 0.00 & 0.04 & 0.21 & 16 & 76 & 0.00 \\
\hline$S 3$ & & 59.5 & 358 & 0.00 & 0.03 & 0.26 & 0.29 & 0.00 & 0.03 & 0.12 & 10 & 90 & 0.00 \\
\hline$S 4$ & & 85.2 & 430 & 0.10 & 2.86 & 6.97 & 7.00 & 0.03 & 2.96 & 0.41 & 41 & 100 & 0.01 \\
\hline S5 & & 71.0 & 427 & 0.00 & 0.16 & 0.83 & 0.36 & 0.00 & 0.16 & 0.19 & 44 & 231 & 0.00 \\
\hline S6 & & 70.8 & 449 & 0.00 & 0.07 & 0.24 & 0.24 & 0.00 & 0.07 & 0.29 & 29 & 100 & 0.00 \\
\hline$S 7$ & & 70.1 & 449 & 0.00 & 0.05 & 0.25 & 0.27 & 0.00 & 0.05 & 0.20 & 19 & 93 & 0.00 \\
\hline
\end{tabular}

method as well as of the interpretation of the results has been presented made by many authors (Peters, 1986; Jarvie, 1991; Peters and Cassa, 1994; Lafargue et al., 1998; Behar et al., 2001; Maravelis and Zelilidis, 2010). In this study, the method of Espitalié et al. (1985) was utilized to evaluate the organic geochemical attributes of the studied rocks. The interpretive guidelines used were from (Tissot and Welte, 1984; Peters, 1986; Peters and Cassa, 1994; Burwood et al., 1995; Dymann et al., 1996).

\section{SOURCE ROCK GEOCHEMISTRY}

Bulk organic geochemical characteristics of the upper Miocene depositional sequence in Messara Basin are summarized in Table 1.

The factors S1, S2, S3, Tmax, HI, OI, PI and TOC were taken into consideration to evaluate the amount, type and maturation of the OM. $S 1$ corresponds to the quantity of free hydrocarbons present in the rock sample (Tyson, 1995). $S 2$ reflects its potential to produce 
hydrocarbons after maturation, while $S 3$ indicates the oxygenated organic compounds content (Tyson, 1995). Tmax is the temperature at which the $S 2$ peak reaches its maximum (Tissot and Welte, 1984; Tyson, 1995). Such factor is used as a maturity parameter for fossil OM (Tissot and Welte, 1984).

Hydrogen Index $(H I)$ reflects the quality and quantity of pyrolyzable organic compounds, from $S 2$ relative to the TOC (mg HC/g TOC). Oxygen Index $(O I)$ is related to the quantity of terrestrial OM. Both parameters define the kerogen type present in the rocks (Tissot and Welte, 1984; Tyson, 1995; Hunt, 1996). Production Index (PI) reveals the total amount of hydrocarbons $(S 1+S 2)$ that may be produced (Jones, 1984). Total Organic Carbon (TOC) provides information about the organic richness in a rock (Tissot and Welte, 1984). It also reveals the sum of organic carbon that is present in the sample and is associated with free hydrocarbons and kerogen (Tissot and Welte, 1984; Jarvie, 1991; Hunt, 1996). The minimum TOC value required to characterize a sample as at least an immature source rock is $0.5 \mathrm{wtH} \%$ (Hunt, 1979; Tissot and Welte, 1984).
In the current research, the definition of the different environments of deposition (marine to lacustrine) on the drilled section was controlled by following the description of Zachariasse et al. (2011).

\subsection{Amount of Organic Matter}

TOC content of the examined samples varies between 0.12 to $7 \mathrm{wtH} \%$ suggesting that the upper Miocene sedimentary succession in Messara Basin may it contains at least immature source rocks. In particular, contain samples which might be of slight interest (20 samples, $0.5-1.0 \%$ ) and samples which are definitely worthy of further consideration (1 sample, > 1.0\%).

Core samples indicate that significant $\mathrm{OM}$ content $($ TOC $>0.5 \%)$ is accumulating in both Viannos and Skinias Fms and forms high TOC intervals (Fig. 5). Compared to the Skinias Fm, the Viannos Fm is with higher organic carbon content and three high TOC intervals have been observed (depth 264-288 m, 334-361 m and $413-445 \mathrm{~m}$ ). These intervals correspond to the mid and upper Viannos Fm and represent a lake

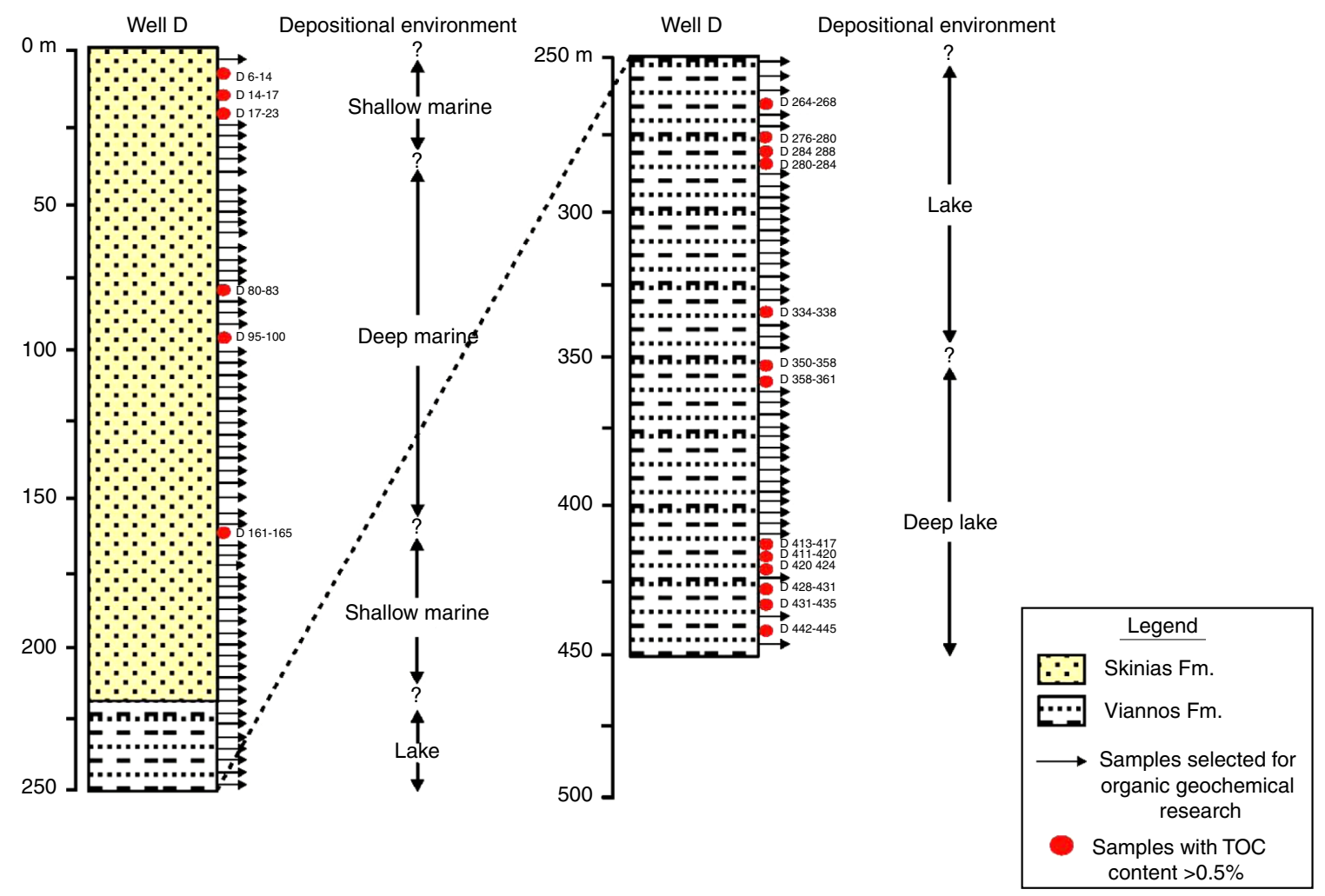

Figure 5

Stratigraphic column that shows the evolution of depositional environments with depth in Well-D. Note the positions of the selected samples (black arrows) and the positions of samples with TOC content $>0.5 \%$ (red cycles). 


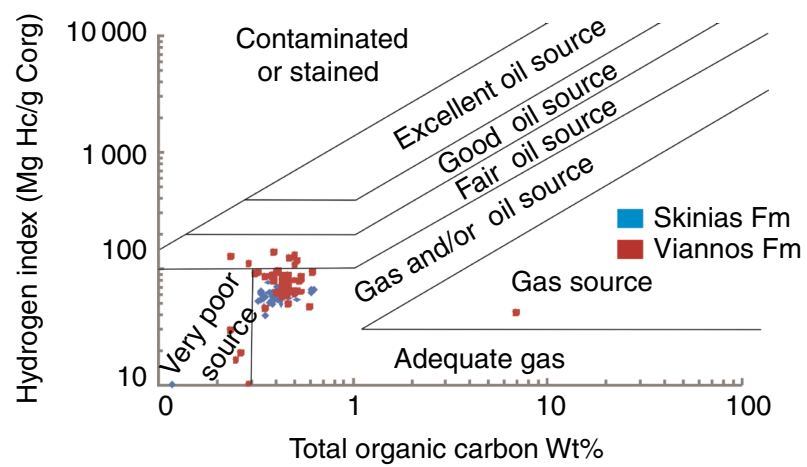

Figure 6

Possible hydrocarbon generation potential of the selected samples using HI versus TOC plot.

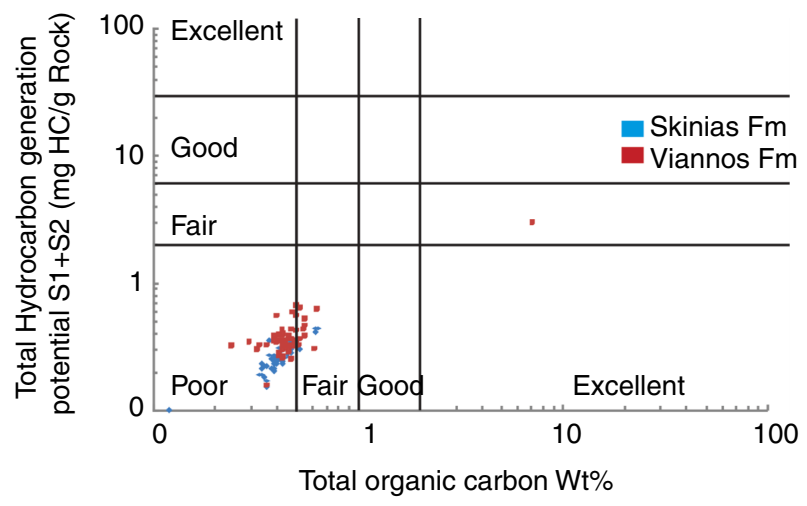

Figure 7

Source rock characterization using plot of total hydrocarbon generation $(S 1+S 2)$ versus total organic carbon (TOC). environment (Fig. 5) (Zachariasse et al., 2011). Skinias Fm has an interval of high interest (depth 6-23) and sparse individual samples with pronounced TOC content (D80-83, D95-100 and D161-165) (Fig. 5). This interval probably corresponds to a shallow-marine environment, typical of the upper Skinias Fm. Likewise, the sparse high TOC samples probably correspond to a deep-marine (D80-83, D95-100) and a shallow-marine (D161-165) environment.

TOC values cannot be used alone as a definite indicator of possible petroleum potential. For example, graphite consisting of essentially $100 \%$ carbon does not generate petroleum. Some tertiary deltaic marine shales contain up to $5 \%$ TOC, practically do not generate petroleum, being gas prone or inert (Peters and Cassa, 1994). A better rating of source rock potential is given by a cross plot of $H I$ versus $T O C$. This diagram indicates hydrocarbon potential of diverse source types, ranging from gas and/or oil to fair oil source (Fig. 6). Nevertheless, with the ratings obtained from these plots it is necessary to integrate the chemistry, with the maturation data and description of OM before a rock can be truly considered as having potential for oil. The ratings however, do give a good indication of the relative hydrocarbon source potential (Jackson et al., 1985).

$S P$ is defined as the sum of $S 1$ and $S 2$ values derived from Rock-Eval pyrolysis. Rocks with $S P>2 \mathrm{HC} / \mathrm{g}$ suggest insignificant oil but some gas potential, rocks with $S P$ ranging between 2 and $6 \mathrm{HC} / \mathrm{g}$ are classified as moderately rich source rocks with fair oil potential and rocks with $S P$ greater that $6 \mathrm{HC} / \mathrm{g}$ are classified as good to excellent source rock potential (Tissot and Welte,
1984; Dymann et al., 1996). Messara Basin contains samples with $S P$ values that range between 0.02 and $2.96 \mathrm{mg} \mathrm{HC} / \mathrm{g}$ rock. Such values are typical of sedimentary rocks with fair/moderate gaseous source rock potential (Fig. 7).

\subsection{Type of Organic Matter}

In this study, kerogen classification diagrams were constructed using the TOC and Rock-Eval analytical data to define the existing kerogen type. $H I$ values $<50 \mathrm{mg}$ $\mathrm{HC} / \mathrm{g}$ TOC imply that the rock contains mainly inert kerogen and values of $>200 \mathrm{mg} \mathrm{HC} / \mathrm{g}$ TOC suggest occurrence of significant amounts of hydrogen-rich (oil prone kerogen) (Tissot and Welte, 1984).

Studied rocks have $H I$ values, which range between 10 (S3) and $135 \mathrm{mg} \mathrm{HC} / \mathrm{g}$ TOC (D445-450) and, in conjunction with Jones (1984) and Bordenave (1993) classification diagram, indicate types III and IV kerogen. Type III kerogen corresponds to partially oxidized terrestrial $\mathrm{OM}$ and type IV to inert OM that contains extremely low amounts of hydrogen (Tissot and Welte, 1984). To further determine the kerogen type, the diagram $\mathrm{HI}-\mathrm{OI}$ was used. On the basis of this diagram, studied rocks contain OM of type III kerogen although samples composed of type IV kerogen also occur. Given the prevalence of type III kerogen, a gaseous hydrocarbon potential is suggested (Fig. 8).

Tmax values vary reflecting the type and maturity of the $\mathrm{OM}$ and, for this reason, HI-Tmax diagram was additionally used to improve our understanding of the kerogen types. This diagram indicates suitability for 
gas production (Type III kerogen) as the principal hydrocarbon potential (Fig. 9). A gaseous generation potential is also indicated by using the $H I$ and $S 2 / S 3$ scheme proposed by Peters (1986) (Tab. 2). Study area contains $\mathrm{OM}$ suitable for gas production $(H I<150 \mathrm{mg} \mathrm{HC} / \mathrm{g}$ Corg., S2/S3<3). The $S 1-T O C$ diagram indicates that the produced hydrocarbons are autochthonous, with no organic contamination and oil generation potential (Fig. 10).

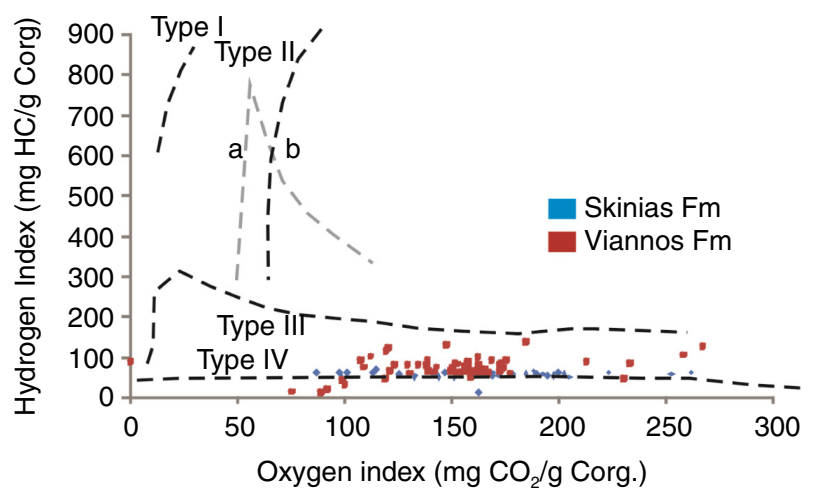

Figure 8

Results of Rock-Eval 2 pyrolysis plotted on a Hydrogen Index $(H I)$ versus Oxygen Index $(O I)$ kerogen classification diagram.

\subsection{Thermal Maturity}

Thermal maturity of the OM can be determined from Rock-Eval analysis results (Tmax, PI) as well as using organic petrographic methods (e.g. spore-color index and vitrinite reflection). An assessment of the degree of thermal maturity reached by the studied Tertiary rocks is provided by Tmax values which vary between $358^{\circ} \mathrm{C}$ and $503^{\circ} \mathrm{C}$ (average $417^{\circ} \mathrm{C}$ ). On the basis of the HI-Tmax diagram, Tmax values suggest that most of the studied sediments are of immature oil stage although samples within the mature oil window occur (Fig. 9). PI values further indicate that a significant number (17) of samples are on the top of the oil window.

PI and Tmax are crude measurements of thermal maturity and are partly dependent on other factors (e.g. the type of OM) (Peters, 1986). The determination of thermal maturity should be supported by others geochemical analyses, such as vitrinite reflectance or Thermal Alteration Index (TAI) (Peters, 1986). Therefore, the results presented in this research comprise a first approach to add information on the regional maturation level. In any case, most of the studied Tertiary rocks are immature with respect to oil generation. They haven't experienced high temperature during burial and most likely contain very little charcoals or recycled material from older mature rocks. Nevertheless, samples of that oil stage have potential to generate gas and the occurrence of gas on Well-D confirms the above statement.

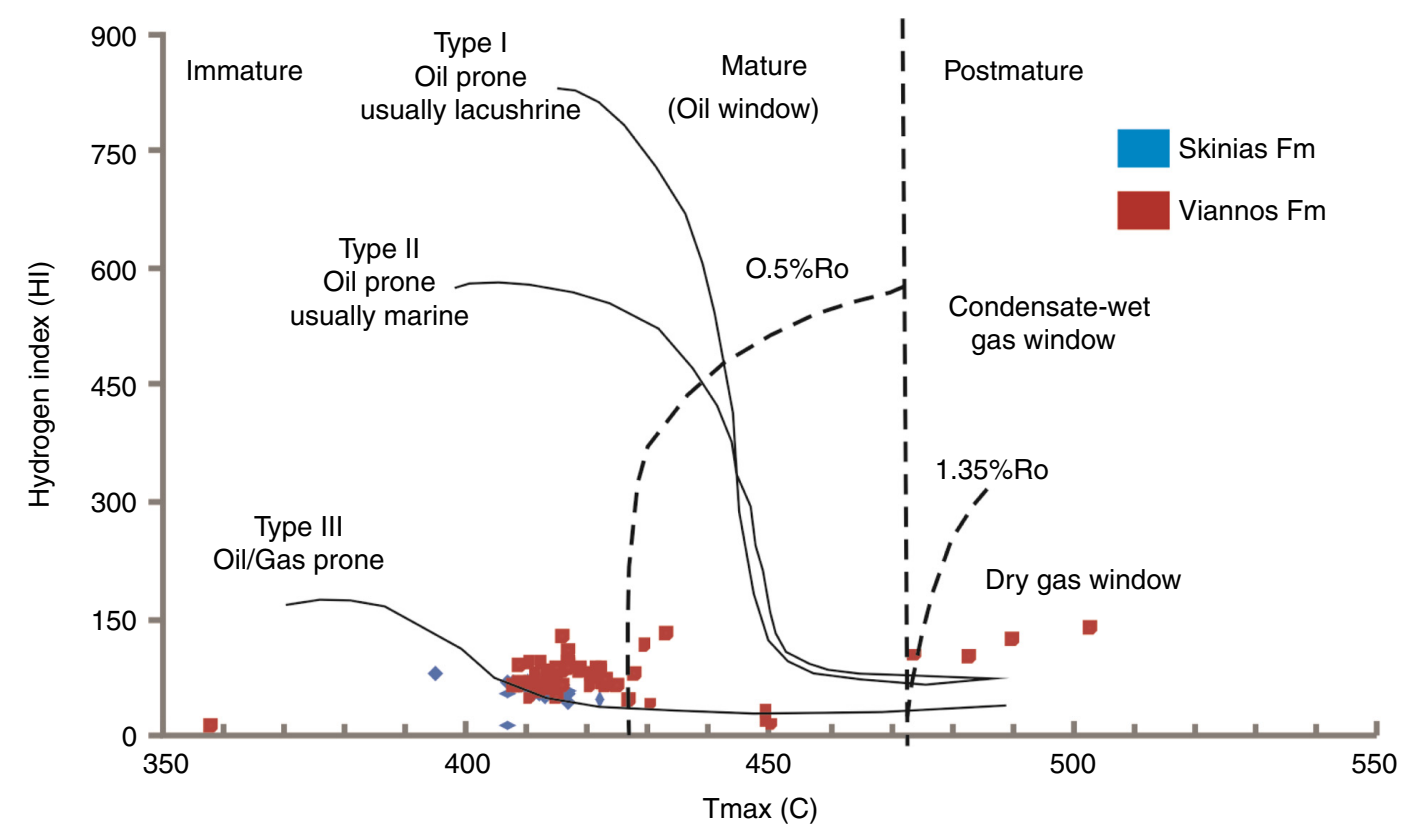

Figure 9

Classification of kerogens of the Late Miocene sediments on Messara Basin using the HI versus Tmax diagram. 
TABLE 2

Geochemical parameters describing type of hydrocarbons generated (from Peters, 1986)

\begin{tabular}{c|c|c}
\hline Type & $\mathrm{HI}\left(\mathrm{mg} \mathrm{HC} / \mathrm{g} \mathrm{C}_{\text {org }}\right)$ & $\mathrm{S} 2 / \mathrm{S} 3$ \\
\hline Gas & $0-150$ & $0-3$ \\
\hline Gas and Oil & $150-300$ & $3-5$ \\
\hline Oil & $300+$ & $5+$ \\
\hline
\end{tabular}

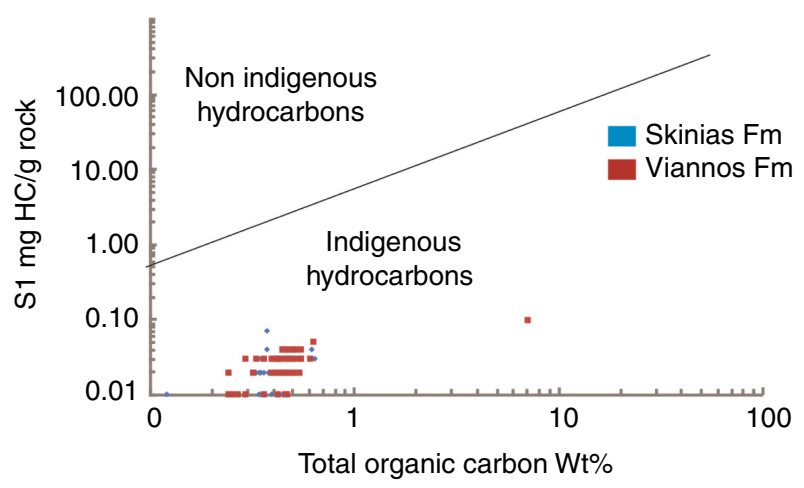

Figure 10

Plot of $S 1$ versus TOC $\mathrm{wt} \%$.

\section{CONCLUSIONS}

Organic geochemical research on Messara Basin, Central Crete, Greece was conducted in order to further evaluate the occurrence of a working petroleum system with source rocks, reservoirs, seals and structures in the region. The research led to establishment of the regional Tertiary gaseous source rock potential and enabled the analysis of its characteristics in terms of the total content of OM, its type, quality and maturity level, together with the analysis of their generative potential. The selected samples are representative of the Viannos and Skinias Fm that comprise the likely regional hydrocarbon source rock facies. These formations were deposited in a wide range of subenvironments including deep lake, lake, shallow and deep-marine depositional settings.

The obtained TOC content suggests that studied sediments appear to be potential source rocks since significant $\mathrm{OM}$ content $($ TOC $>0.5 \%)$ is accumulating in both formations. Viannos Fm is with higher values and contains three of high TOC intervals. Such intervals occur in the mid and upper parts of the formation and correspond to a lake environment. Skinias Fm has an interval of high interest in conjunction with sparse, individual samples with high TOC content. The higher TOC interval is observed in upper parts of the Skinias Fm and probably corresponds to a shallow-marine environment. The remaining high TOC samples most likely correspond to a deep and a shallow-marine environment.

The second prerequisite for good petroleum source rock is the quality of the OM. Rock Eval II parameters indicates that the Messara Basin contains source rocks with gas-prone hydrocarbon potential. Although the sedimentary overburden was not sufficient to reach the beginning of the oil window in this part of the basin, these rocks and the lower Viannos Fm can be considered as potential source rocks. The channel-belt/overbank deposits associated with sandy reservoirs, in different parts of the basin with higher thermal evolution, may have already reached the oil window.

Bearing in mind that on study area the four magic ingredients namely source, reservoir, trap and seal appear to occur, Greece should proceed with additional extensive geological studies and detailed geological surveys to explore the existence of hydrocarbons offshore south, southeast and southwest of Crete.

\section{ACKNOWLEDGMENTS}

The work presented in this article presents the research conducted by the Department of Geology, University of Patras, Greece and the Hydrocarbon Chemistry and Technology Research Unit, Technical University of Crete, Chania, Greece. Funding was supported by the Greek Secretariat for Research and Technology and local authorities. The authors would like to acknowledge Professor A. Pavlopoulos (Univ. Athens) for his thorough editing and suggested changes to French.

\section{REFERENCES}

Angelier J., Lyberis N., Le Pichon X., Barrier P., Huchon P. (1982) The tectonic development of the Hellenic arc and the Sea of Crete: a synthesis, Tectonophysics 86, 159-196.

Behar F., Beaumont V.De.B., Penteado H.L. (2001) Rock-Eval 6 technology: performances and developments, Oil Gas Sci, Technol. 56, 111-134.

Bordenave M.L. (1993) Applied petroleum geochemistry, in Exploration Division TOTAL, Bordenave M.L. (ed.), Paris.

Bruneton A., Konofagos E., Foscolos A. (2011) Economic and Geopolitical Importance of Eastern Mediterranean gas fields for Greece and the E. U. Emphasis on the Probable Natural Gas Deposits Occurring in the Libyan Sea within the Exclusive Economic Zone of Greece, Mineral Wealth 160, 7-30. 
Burwood R., De Witte S.M., Mycke B., Paulet J. (1995) Petroleum geochemical characterization of the lower Congo Coastal Basin Bucomazi formation, in Petroleum Source Rocks, Katz B.J. (ed.), Springer-Verlag, Berlin.

Camerlenghi A., McCoy W.F. (1990) Physiography and structure of Bacino Bannock (Eastern Mediterranean), Geo-Marine Lett. 10, 1, 15-23.

Chatzaras V., Xypolias P., Doutsos T. (2006) Exhumation of high-pressure rocks under continuous compression: a working hypothesis for the southern Hellenides (central Crete, Greece), Geol. Mag. 143, 859-876.

Cita M.B. (1991) Anoxic basins of the eastern Mediterranean: An overview, Paleoceanography 6, 1, 133-141.

Dymann T.S., Palacos J.G., Tysdal R.G., Perry W.J., Pawlewicz M.J. (1996) Source rock potential of middle cretaceous rocks in Southwestern Montana, Am. Assoc. Pet. Geol. Bull. 80, 1177-1184.

Emery K.O., Heezen B., Allan T.D. (1966) Bathymetry of the Eastern Mediterranean sea, Deep Sea Res. 13, 173-192.

Espitalié J., Laporte J.L., Madec M., Marquis F., Leplat P., Paulet J. (1977) Méthode rapide de caractérisation des roches mères, de leur potentiel pétrolier et de leur degré dévolution, Rev. Inst. Fr. Petr. 32, 23-45.

Espitalié J., Marquis F., Barsony I. (1985) Geochemical logging, in Analytical Pyrolysis-Techniques and Applications, Voorhees K.J. (ed.), Boston, Butterworth.

Faccenna C., Jolivet L., Piromallo C., Morelli A. (2003) Subduction and the depth of convection of the Mediterranean mantle, J. Geophys. Res. 108, 2099.

Fassoulas C. (2001) The tectonic development of a Neogene basin at the leading edge of the active European margin: the Heraklion basin, Crete, Greece, J. Geodynamics 31, 49-70.

Gardosh M.A., Druckman Y., Binyamin Buchbinder B. (2009) The Late Tertiary Deep-Water Siliciclastic System of the Levant Margin-An Emerging Play Offshore Israel, $A A P G$ Convention, Denver, Colorado.

Heezen B.C., Ewing M. (1963) The Mid Oceanic Ridge, in The Seas Interscience, Hill M.N. (ed.), New York.

Hersey J.B. (1965) Sedimentary basins of the Mediterranean Sea, in Submarine Geology and Geophysics, in $17^{\text {th }}$ Symposium Colston Res, Soc., 5-9 April, Whittard W.F., Bradshaw R. (eds.), Butterworths, London.

Huchon P., Lyberis N., Angelier J., Le Pichon X., Renard V. (1982) Tectonics of the Hellenic Trench: a synthesis of Sea-Beam and submersible observations, Tectonophysics 86, 69-112.

Huguen C., Mascle J. (2001) La Marge Lybienne, entre $23^{\circ} 30$ et $25^{\circ} 30$ de Longitude Est, Comptes Rendus de l'Academie des Sciences de Paris 332, 553-560.

Hunt J.M. (1979) Petroleum Geochemistry and Geology, Freeman and Company, San Francisco.

Hunt J.M. (1996) Petroleum Geochemistry and Geology, Freeman and Company, New York.

Jackson K.S., Hawkins P.J., Bennett A.J.R. (1985) Regional facies and geochemical evaluation of Southern Denison Trough, APEA J. 20, 143-158.

Jacobshagen V. (1986) Geologie Von Griechenland, Borntraeger, Berlin-Stuttgart.

Jarvie D.M. (1991) Factors Affecting Rock-Eval Derived kinetic parameter, Chem. Geol. 93, 79-99.
Jones R.W. (1984) Comparison of carbonate and shale source rocks, in Petroleum Geochemistry and Source Potential of Carbonate Rocks, Palacas J. (ed.), AAPG Stud. Geol.

Kreemer C., Chamot-Rooke N. (2004) Contemporary kinematics of the southern Aegean and the Mediterranean Ridge, Geophys. J. Int. 157, 1377-1392.

Krois P., Hanke K., Novotny B., Bayoumi T., Hussein H., Tari G. (2010) The emerging deepwater Province of Northwest Egypt, AAPG International Conference, Rio de Janeiro, Brazil.

Lafargue E., Marquis F., Pillot D. (1998) Rock-Eval 6 applications in hydrocarbon exploration, production, and soil contamination studies, Oil Gas Sci. Technol. 53, 421-437.

Maravelis A., Zelilidis A. (2010) Organic geochemical characteristics of the late Eocene-early Oligocene submarine fans and shelf deposits on Lemnos Island, NE Greece, J. Petrol. Sci. Eng. 71, 160-168.

Maravelis A., Manutsoglu E., Konstantopoulos P., Pantopoulos G., Makrodimitras G., Zoumpouli E., Zelilidis A. (2012) Hydrocarbons plays and prospectivity of the Mediterranean Ridge, Energy Sources, Part A: Recovery, Utilization, and Environmental Effects (in press).

Meulenkamp J.E., Sissingh W. (2003) Tertiary palaeogeography and tectonostratigraphic evolution of the Northern and Southern Peri-Tethys platforms and the intermediate domains of the African-Eurasian convergent plate boundary zone, Palaeogeogr. Palaeoclimatol. Palaeoecol. 196, 1-2, 209-228.

Meulenkamp J.E., Georgiadou-Dikeoulia E., Jonkers H.A., Boger H. (1979) Field guide to the Neogene of Crete, Publ. Dept. Geol. Paleont. Athens Univ. 32, 1-32.

Meulenkamp J.E., van der Zwaan G.J., Van Wamel W.A. (1994) On Late Miocene to Recent vertical motions in the Cretan segment of the Hellenic arc, Tectonophysics 234, 53-72.

Montadert L., Nikolaides S. (2007) The Geological structure of the Eratosthenes Continental Block and its Margins with the Levantine and Herodotus Basins (Eastern Mediterranean) from New Seismic Reflection Data, AAPG European Region Conference, Athens, Greece.

Olivet J.L., Bonnin J., Beuzart P., Auzende J.M. (1982) Cinématique des plaques et paléogeographie: une revue, Geol. Soc. Fr. Bull. 24, 875-892.

Panagopoulos G., Hamdan H., Spyridonos E., Pyliotis I., Zelilidis A., Vafidis A., Manoutsoglou E. (2011a) 3D Geological Modeling using VES Data. An Application on MethaneBearing Neogene Deposits at Arkalochori Region, Messara, Proceedings of BGS Congress, Budapest.

Panagopoulos G., Pyliotis I., Zelilidis A., Spyridonos E., Hamdan H., Vafidis A., Manoutsoglou E. (2011b) 3D modeling of biogenic gas-bearing Neogene deposits at Arkalochori region, Messara, Crete, Greece, Proceedings of IAMG, Saltzburg 431-440.

Papanikolaou D., Vassilakis E. (2010) Thrust faults and extensional detachment faults in Cretan tectono-stratigraphy: Implications for Middle Miocene extension, Tectonophysics 488, 233-247.

Peters K.E. (1986) Guidelines for evaluating petroleum source rocks using programmed pyrolysis, American Association of Petroleum Geologists Bulletin 70, (3), 319-329.

Peters K.E., Cassa M.R. (1994) Applied source geochemistry, in The Petroleum System-From Source to Trap, Magoon L. B., Dow W.G. (eds.), AAPG Bull. 
Postma G., Fortuin A.R., Van Wamel W.A. (1993) Basin-fill patterns controlled by tectonics and climate: the Neogene "fore-arc" basins of eastern Crete as a case history, Publ. Int. Assoc. Sediment. 20, 335-362.

Reillinger R.E., McClusty S.C., Oral M.B., King R.W., Toksoz M.N. (1997) Global positionning system measurements of present-day crustal movements in the Arabia-Africa-Eurasia plate collision zone, J. Geophys. Res. 102, 9983-9999.

Reuter M., Brachert T.C. (2007) Freshwater discharge and sediment dispersal-control on growth, ecological structure and geometry of Late Miocene shallow-water coral ecosystems (Early Tortonian, Crete/Greece), Palaeogeogr. Palaeoclimatol. Palaeoecol. 255, 3-4, 308-328.

Reuter M., Brachert T.C., Kroeger K.F. (2006) Shallowmarine carbonates of the tropical-temperate transition zone: effects of hinterland climate and basin physiography (Late Miocene, Crete/Greece) Pedley H.M., Carannante S. (eds), Cool-Water Carbonates, Geol. Soc. London, Spec. Publ. 255, 157-178.

Ring U., Brachert T., Fassoulas C. (2001) Middle Miocene graben development in Crete and its possible relation to largescale detachment faults in the southern Aegean, Terra Nova 13, 297-304.

Rögl F., Steininger F. (1984) Neogene Paratethys, Mediterranean and Indo-Pacific seaways: implications for the paleobiogeography of marine and terrestrial biotas, in Fossils and climate, Brenchley P. (ed.), New York, Wiley.

Ryan W.B.F., Stanley D.J., Hersey J.B., Fahlquist D.A., Allan T.D. (1971) The tectonics and geology of the Mediterraneran Sea, The Sea, Maxwell A.E. (ed.), New York, Wiley-Interscience.

Semb H.P. (2009) Possible seismic hydrocarbon indicators in the offshore Cyprus and Lebanon, GeoArabia 14, 2, 49-66.

Ten Veen J.H., Meijer P.Th. (1998) Late Miocene to Recent tectonic evolution of Crete (Greece): geological observations and model analysis, Tectonophysics 298, 191-208.
Ten Veen J.H., Postma G. (1999) Neogene tectonics and basin fill patterns in the Hellenic outer-arc (Crete, Greece), Basin Res. 11, 223-242.

Tissot B.P., Welte D.H. (1984) Petroleum formation and occurrence, Springer-Verlag.

Tortorici L., Caputo R., Monaco C. (2010) Late Neogene to Quaternary contractional structures in Crete (Greece), Tectonophysics 483, 203-213.

Tyson R.V. (1995) Sedimentary organic matter, organic facies and palynofacies, Chapman and Hall, London.

Van Hinsbergen D.J.J., Hafkenscheid E., Spakman W., Meulenkamp J.E., Wortel M.J.R. (2005) Nappe stacking resulting from subduction of oceanic and continental lithosphere below Greece, Geology 33, 325-328.

Van Hinsbergen D.J.J., Meulenkamp J.E. (2006) Neogene supradetachment basin development on Crete (Greece) during exhumation of the South Aegean core complex, Basin Res. 18, 103-124.

Winkler S., Haakensen N., Nesje A., Rye N. (1997) Glaziale Dynamik in Westnorwegen - Ablauf und Ursachen des aktuellen Gletschervorstoßes am Jostedalsbreen, Petermanns Geographische Mitteilungen 141, 43-63.

Zachariasse W.J., Van Hinsbergen D.J.J., Fortuin A.R. (2011) Formation and fragmentation of a late Miocene supradetachment basin in central Crete: implications for exhumation mechanisms of high-pressure rocks in the Aegean forearc, Basin Res. 23, 6, 678-701.

Cite this article as: A. Maravelis, G. Panagopoulos, I. Piliotis, N. Pasadakis, E. Manoutsoglou and A. Zelilidis (2013). Pre-Messinian (Sub-Salt) Source-Rock Potential on Back-Stop Basins of the Hellenic Trench System (Messara Basin, Central Crete, Greece), Oil Gas Sci. Technol 71, 6. 